\title{
THE SIZE OF EXPONENTIAL SUMS ON INTERVALS OF THE REAL LINE
}

\author{
Tamás ERdélyi, KaVeh Khodjasteh, and Lorenza Viola
}

Abstract. We prove that there is a constant $c>0$ depending only on $M \geq 1$ and $\mu \geq 0$ such that

$$
\int_{y}^{y+a}|g(t)| d t \geq \exp (-c /(a \delta)), \quad a \delta \in(0, \pi]
$$

for every $g$ of the form

$$
g(t)=\sum_{j=0}^{n} a_{j} e^{i \lambda_{j} t}, \quad a_{j} \in \mathbb{C},\left|a_{j}\right| \leq M j^{\mu},\left|a_{0}\right|=1, n \in \mathbb{N},
$$

where the exponents $\lambda_{j} \in \mathbb{R}$ satisfy

$$
\lambda_{0}=0, \quad \lambda_{j} \geq j \delta>0, \quad j=1,2, \ldots,
$$

and for every subinterval $[y, y+a]$ of the real line. Establishing inequalities of this variety is motivated by problems in physics.

\section{INTRODUCTION}

The well known Littlewood conjecture was solved by Konyagin [8] and independently by McGehee, Pigno, and B. Smith [10]. Based on these Lorentz [5] worked out a textbook proof of the conjecture.

Theorem 0.1. There is an absolute constant $c>0$ such that

$$
\int_{0}^{2 \pi}\left|\sum_{j=1}^{n} e^{i \lambda_{j} t}\right| d t \geq c \log n
$$

whenever $\lambda_{1}, \lambda_{2}, \ldots, \lambda_{n}$ are distinct integers.

This is an obvious consequence of the following result.

Key words and phrases. exponential sums, constrained coefficients, subarcs, unit circle, Littlewood's conjecture, Konyagin's conjecture, Uhrig protocol, decoupling methods, quantum coherence, multi-pulse control.

2000 Mathematics Subject Classifications. Primary: 11L03, 11L07, 41A10, 41A17, Secondary: $41 \mathrm{~A} 05$ 
Theorem 0.2. Let $\lambda_{1}<\lambda_{2}<\cdots<\lambda_{n}$ be integers. Let $a_{1}, a_{2}, \ldots, a_{k}$ be arbitrary complex numbers. We have

$$
\int_{0}^{2 \pi}\left|\sum_{j=1}^{n} a_{j} e^{i \lambda_{j} t}\right| d t \geq \frac{1}{30} \sum_{j=1}^{n} \frac{\left|a_{j}\right|}{j} .
$$

Pichorides, who contributed essentially to the proof of the Littlewood conjecture, observed in [11] that the original Littlewood conjecture (when all the coefficients are from $\{0,1\}$ ) would follow from a result on the $L_{1}$ norm of such polynomials on sets $E \subset \partial D$ of measure $\pi$. Namely if

$$
\int_{E}\left|\sum_{j=0}^{n} z^{\lambda_{j}}\right||d z| \geq c
$$

for any subset $E \subset \partial D$ of measure $\pi$ and for any nonnegative integers $\lambda_{0}<\lambda_{1}<\cdots<\lambda_{n}$ with an absolute constant $c>0$, then the original Littlewood conjecture holds. Here $\partial D$ denotes the unit circle of the complex plane and the measure of a set $E \subset \partial D$ is the linear Lebesgue measure of the set

$$
\left\{t \in[-\pi, \pi): e^{i t} \in E\right\} .
$$

Konyagin [9] gives a lovely probabilistic proof showing that this hypothesis fails. He does however conjecture the following: for any fixed set $E \subset \partial D$ of positive measure there exists a constant $c=c(E)>0$ depending only on $E$ such that

$$
\int_{E}\left|\sum_{j=0}^{n} z^{\lambda_{j}}\right||d z| \geq c(E)
$$

for any nonnegative integers $\lambda_{0}<\lambda_{1}<\cdots<\lambda_{n}$. In other words, the sets $E_{\varepsilon} \subset \partial D$ of measure $\pi$ in his example where

$$
\int_{E_{\varepsilon}}\left|\sum_{j=0}^{n} z^{\lambda_{j}}\right||d z|<\varepsilon
$$

must vary with $\varepsilon>0$.

In [2] we show, among other things, that Konyagin's conjecture holds on subarcs of the unit circle $\partial D$.

In [7] S. Güntürk constructs certain types of near-optimal approximations of a class of analytic functions in the unit disk by power series with two distinct coefficients. More precisely, it is shown that if all the coefficients of the power series $f(z)$ are real and lie in $[-\mu, \mu]$, where $\mu<1$, then there exists a power series $Q(z)$ with coefficients in $\{-1,+1\}$ such that $|f(z)-Q(z)| \rightarrow 0$ at the rate $\exp \left(C|1-z|^{-1}\right)$ as $z \rightarrow 1$ non-tangentially inside the unit disk. Güntürk refers to P. Borwein, Erdélyi, and Kós in [4] to see that this type of decay rate is best possible. The special case $f \equiv 0$ yields a near-optimal solution to the "fair duel problem" of Konyagin, as it is described in the Introduction of [7]. form

In this paper we extend the polynomial inequalities of [2] to exponential sums of the

$$
g(t)=\sum_{j=0}^{n} a_{j} e^{i \lambda_{j} t}, \quad a_{j} \in \mathbb{C},\left|a_{j}\right| \leq M j^{\mu},\left|a_{0}\right|=1, n \in \mathbb{N},
$$


where $\lambda_{0}=0$ and the real exponents satisfy the "minimum growth condition"

$$
\lambda_{j} \geq j \delta>0, \quad j=1,2, \ldots
$$

In addition to being interesting on its own, this extension is motivated by physical applications in the context of decoherence control in open quantum systems using dynamical decoupling methods [6]. In the paradigmatic case of a single two-level quantum system undergoing pure dephasing due to coupling to a quantum bosonic environment, for instance, the residual decoherence error at a time $t$ after the application of $n$ ideal "spin-flip" pulses at times $0<t_{1}<t_{2}<\ldots<t_{n}<T$, is quantified by a decay factor of the form

$$
\chi_{\left\{t_{j}\right\}}=\int_{0}^{\infty} \Lambda(\omega)\left|f_{\left\{t_{j}\right\}}(\omega)\right|^{2} d \omega, \quad f_{\left\{t_{j}\right\}}(\omega)=\sum_{j=0}^{n}(-1)^{j}\left(e^{i t_{j} \omega}-e^{i t_{j+1} \omega}\right),
$$

where $\Lambda(\omega)$ is a real function whose details depend on both the temperature and the density of modes at frequency $\omega$ in the environment and, in addition, $t_{0}:=0$ and $t_{n+1}:=T$. Thus, the decoherence error corresponds directly to the size of the exponential sum $f_{\left\{t_{j}\right\}}(\omega)$.

Decoupling methods aim to design the "filter function" $f_{\left\{t_{j}\right\}}(\omega)$ in such a way that the error $\chi_{\left\{t_{j}\right\}}$ is minimized [13]. Under the assumption that the spectral density of the environment (hence $\Lambda(\omega)$ ) vanishes for frequencies higher than an "ultraviolet cut-off frequency" $\omega_{c}$, the decay factor $\chi_{\left\{t_{j}\right\}}$ may be made small by requiring the exponential sum to vanish perturbatively, i.e., to start its Taylor series at a sufficiently high order $\left(\omega_{c} T\right)^{m}$. In particular, it has been recently shown [12] that if the pulse timings are chosen according to $t_{j}=T \sin ^{2}(j \pi /(2 n+2))$, cancellation of $\chi_{\left\{t_{j}\right\}}$ is achieved to order $m=n$ by using $n$ pulses, the so-called Uhrig decoupling. Physically, however, a minimum growth condition is always imposed by the fact that the separation between any two consecutive pulses cannot be made arbitrarily small due to finite timing resources, thus $t_{j+1}-t_{j}>\tau>0$ for all $j$. As shown in [6], the results established here may then be used to obtain a nonperturbative lower bound on $\chi_{\left\{t_{j}\right\}}$, determined solely in terms of the parameter $\omega_{c} \tau$. As an additional implication of our analysis, we find that Uhrig decoupling arises naturally as a consequence of representing certain polynomials of degree at most $2 n+1$ in terms of Lagrange interpolation at the extreme points of the Chebyshev polynomials $U_{2 n+1}$.

\section{Notation}

For $N>0$ and $\mu \geq 0$, let $\mathcal{S}_{N}^{\mu}$ denote the collection of all analytic functions $f$ on the open unit half-disk $D^{+}:=\{z \in \mathbb{C}:|z|<1, \operatorname{Re}(z)>0\}$ that satisfy

$$
|f(z)| \leq \frac{N}{(1-|z|)^{\mu}}, \quad z \in D^{+} .
$$

In this note the value of $\mu$ will always assumed to be a nonnegative integer. We define the following subsets of $\mathcal{S}_{1}^{1}$. Let

$$
\mathcal{F}_{n}:=\left\{f: f(x)=\sum_{j=0}^{n} a_{j} x^{j}, a_{j} \in\{-1,0,1\}\right\}
$$


and denote the set of all polynomials with coefficients from the set $\{-1,0,1\}$ by

$$
\mathcal{F}:=\bigcup_{n=0}^{\infty} \mathcal{F}_{n}
$$

More generally we define the following classes of Müntz polynomials. For $M>0, \mu \geq 0$, and a sequence $\Lambda:=\left(\lambda_{j}\right)_{j=0}^{\infty}$ of real numbers let

$$
\mathcal{K}_{M}^{\mu}(\Lambda):=\left\{f: f(x)=\sum_{j=0}^{n} a_{j} x^{\lambda_{j}}, a_{j} \in \mathbb{C},\left|a_{j}\right| \leq M j^{\mu},\left|a_{0}\right|=1, n \in \mathbb{N}\right\} .
$$

Here we define the analytic function $z^{\lambda_{j}}:=\exp \left(\lambda_{j} \log z\right)$ by taking the principal analytic branch of $\log z$ in $\mathbb{C} \backslash(-\infty, 0]$.

\section{New Results}

Theorem 2.1. There is a constant $c>0$ depending only on $M \geq 1$ and $\mu \geq 0$ such that

$$
\int_{y}^{y+a}|g(t)| d t \geq \exp (-c /(a \delta)), \quad a \delta \in(0, \pi],
$$

for every $g$ of the form

$$
g(t)=\sum_{j=0}^{n} a_{j} e^{i \lambda_{j} t}, \quad a_{j} \in \mathbb{C},\left|a_{j}\right| \leq M j^{\mu},\left|a_{0}\right|=1, n \in \mathbb{N},
$$

where the exponents $\lambda_{j} \in \mathbb{R}$ satisfy

$$
\lambda_{0}=0, \quad \lambda_{j} \geq j \delta>0, \quad j=1,2, \ldots,
$$

and for every subinterval $[y, y+a]$ of the real line.

Using the substitution $u=t / \delta-y-a / 2$ we need to prove Theorem 2.1 only in the case when $[y, y+a]=[-a / 2, a / 2]$ and $\delta=1$. Hence, using the substitution $z=e^{i t}$, we need to prove only the following result.

Theorem 2.2. There is a constant $c>0$ depending only on $M \geq 1$ and $\mu \geq 0$ such that

$$
\int_{A}|f(z)||d z| \geq \exp (-c / a), \quad a \in(0, \pi]
$$

for every $f$ of the form

$$
f(z)=\sum_{j=0}^{n} a_{j} z^{\lambda_{j}}, \quad a_{j} \in \mathbb{C},\left|a_{j}\right| \leq M j^{\mu}, \quad\left|a_{0}\right|=1, n \in \mathbb{N},
$$


where the exponents $\lambda_{j} \in \mathbb{R}$ satisfy

$$
\lambda_{0}=0, \quad \lambda_{j} \geq j>0, \quad j=1,2, \ldots,
$$

and for every subarc $A:=\left\{e^{i t}: t \in[-a / 2, a / 2]\right\}$ of the unit circle.

Remark 2.3. Without a growth condition on the exponents $\lambda_{j}$ a sequence $\left(g_{k}\right)$ of exponential sums

$$
g_{k}(t)=\sum_{j=0}^{n_{k}} a_{j, k} e^{i \lambda_{j} t}, \quad a_{j, k} \in \mathbb{C},\left|a_{j, k}\right| \leq 1, \quad\left|a_{0, k}\right|=1,
$$

can certainly converge to 0 uniformly on any fixed interval $[-a, a]$. This can easily be seen by taking

$$
\lambda_{0}:=0, \quad \lambda_{j}:=2 / j, \quad j=1,2, \ldots,
$$

and the two term exponential sums

$$
g_{k}(t):=1-\exp \left(i \lambda_{k} t\right) .
$$

Clearly,

$$
\lim _{k \rightarrow \infty} \max _{[-a, a]}\left|g_{k}(t)\right|=\lim _{k \rightarrow \infty} 2 \sin (a / k)=0 .
$$

Remark 2.4. The lower bounds in Theorems 2.1 and 2.2 cannot be essentially improved even if we assume that the exponents

$$
0=\lambda_{0}<\lambda_{1}<\lambda_{2}<\cdots
$$

are integers and $a_{j} \in\{-1,1\}$ for each $j$. Namely, in [2] the authors proved that there are absolute constants $c_{1}>0$ and $c_{2}>0$ such that

$$
\inf _{0 \neq f \in \mathcal{F}} \max _{z \in A}|f(z)| \leq \exp \left(-c_{1} / a\right)
$$

whenever $A$ is a subarc of the unit circle with arclength $\ell(A)=a \leq c_{2}$.

Remark 2.5. An explicit construction showing that Theorems 2.1 and 2.2 cannot be essentially improved can be given by utilizing the fact that if $n$ is even then

$$
f_{n}(z):=-z+1+2 \sum_{k=1}^{n}(-1)^{k} z^{d_{k}}, \quad d_{k}:=\sin ^{2}\left(\frac{k \pi}{2 n+2}\right),
$$

has a zero at 1 with multiplicity at least $n+1$. Namely we prove that there is an absolute constant $c>0$ such that

$$
\inf _{g} \max _{t \in[-a, a]}|g(t)| \leq 12 \exp (-c / a)
$$


for all $a \in(0,1 / 3]$, where the infimum is taken for all exponential sums $g$ of the form

$$
g(t)=\sum_{k=0}^{n+1} a_{k} e^{i \lambda_{k} t}
$$

with $0=\lambda_{0}<\lambda_{1}<\cdots<\lambda_{n+1}$ satisfying the gap condition

$$
\lambda_{k+1}-\lambda_{k} \geq 1, \quad k=0,1, \ldots, n,
$$

$(n=2,4, \ldots$ can be arbitrary) and with

$$
a_{0}=1, \quad a_{n+1}=-1, \quad a_{k}=(-1)^{k} 2, \quad k=1,2, \ldots, n .
$$

Note that in the context of dynamical decoupling theory, the exponents $d_{k}$ are the relative timings of the Uhrig protocol with $n$ pulses [12]. To see that $g_{n}$ has a zero at 1 with multiplicity at least $n+1$, observe that the Lagrange interpolation formula associated with $2 n+1$ distinct points (see [3, p. 8]) reproduces any polynomial of degree at most $2 n$. In particular, choosing the nodes to be the zeros

$$
\alpha_{k}:=\cos \left(\frac{k \pi}{2 n+2}\right), \quad 1 \leq k \leq 2 n+1,
$$

of the Chebyshev polynomial $U_{2 n+1}$ (see [3, Section 2.1] about some of the basic facts about Chebyshev polynomials including the symmetry of the their zeros

$$
\alpha_{2 n+2-k}=-\alpha_{k}, \quad k=1,2, \ldots, n,
$$

and $\left.\alpha_{m+1}=0\right)$, we deduce that

$$
\begin{aligned}
Q(x) & =\sum_{k=1}^{2 n+1} Q\left(\alpha_{k}\right) \frac{U_{2 n+1}(x)}{U_{2 n+1}^{\prime}\left(\alpha_{k}\right)\left(x-\alpha_{k}\right)} \\
& =\sum_{k=1}^{2 n+1} Q\left(\alpha_{k}\right) \frac{(-1)^{k+1}\left(1-\alpha_{k}^{2}\right) U_{2 n+1}(x)}{(2 n+2)\left(x-\alpha_{k}\right)} \\
& =Q(0) \frac{-U_{2 n+1}(x)}{(2 n+2) x}+\sum_{k=1}^{n} Q\left(\alpha_{k}\right) \frac{(-1)^{k+1}\left(1-\alpha_{k}^{2}\right) 2 x U_{2 n+1}(x)}{(2 n+2)\left(x^{2}-\alpha_{k}^{2}\right)}
\end{aligned}
$$

hence

$$
Q(1)=Q(0)+2 \sum_{k=1}^{n} Q\left(\alpha_{k}\right) \frac{(-1)^{k+1} U_{2 n+1}(1)\left(1-\alpha_{k}^{2}\right)}{(2 n+2)\left(1-\alpha_{k}^{2}\right)}=1+2 \sum_{k=1}^{n}(-1)^{k+1} Q\left(\alpha_{k}\right)
$$

for every polynomial $Q$ of degree at most $2 n+1$. Here we used that

$$
\alpha_{2 n+2-k}=-\alpha_{k},{ }_{6} \quad k=1,2, \ldots, n,
$$


and $\alpha_{n+1}=0$. Choosing

$$
Q(x):=\left(1-x^{2}\right)^{m}, \quad m=1,2, \ldots, n,
$$

we obtain

$$
0=1+2 \sum_{k=1}^{n}(-1)^{k+1}\left(1-\alpha_{k}^{2}\right)^{m}, \quad m=1,2, \ldots, n
$$

That is,

$$
0=-1+2 \sum_{k=1}^{n}(-1)^{k} d_{k}^{m}, \quad m=1,2, \ldots, n
$$

while the assumption that $n$ is even yields

$$
0=\sum_{k=1}^{n}(-1)^{k} d_{k}^{m}, \quad m=0
$$

Thus,

$$
f_{n}^{(m)}(1)=0, \quad m=0,1, \ldots, n,
$$

indeed. Now let

$$
g_{n}(t):=f_{n}\left(e^{i t}\right)
$$

Then

$$
g_{n}^{(m)}(0)=0, \quad m=0,1, \ldots, n,
$$

and

$$
\left|g_{n}^{(m)}(u)\right|=\left|-i^{m} e^{i u}+2 \sum_{k=1}^{n}(-1)^{k}\left(i d_{k}\right)^{m} e^{i d_{k} u}\right| \leq 2 n+1, \quad u \in \mathbb{R}, \quad m=1,2,3, \ldots
$$

Let $b=\frac{3}{n+1}$. By using the integral form of the well known Taylor's Remainder Theorem we have

$$
\begin{aligned}
\left|g_{n}(t)\right| & =\left|\frac{1}{n !} \int_{0}^{t} g_{n}^{(n+1)}(u)(t-u)^{n} d u\right| \\
& \leq \frac{1}{n !} \int_{0}^{t}\left|g_{n}^{(n+1)}(u)(t-u)^{n}\right| d u \\
& \leq \frac{1}{(n+1) !} \max _{u \in[-|t|,|t|]}\left|g_{n}^{(n+1)}(u)\right||t|^{n+1} \leq\left(\frac{e t}{n+1}\right)^{n+1}(2 n+1) \\
& \leq \frac{6}{b}\left(\frac{e}{3}\right)^{3 / b}
\end{aligned}
$$

whenever $|t| \leq 1 / b$. (Note that the integral form of Taylor's Remainder Theorem is valid for complex-valued functions, so we do not need to separate the real and imaginary parts 
of the function $g_{n}$ to apply it.) Now with $\lambda_{0}:=0, \lambda_{n+1}:=9 / b^{2}$, and $\lambda_{k}:=\left(9 / b^{2}\right) d_{k}, k=$ $1,2, \ldots, n$, let

$$
G_{b}(t):=g_{n}\left(9 t / b^{2}\right)=-e^{i \lambda_{n+1} t}+e^{i \lambda_{0} t}+2 \sum_{k=1}^{n}(-1)^{k+1} e^{i \lambda_{k} t}
$$

Elementary calculus shows that the exponents $\lambda_{k}$ satisfy the gap condition

$$
\begin{aligned}
\lambda_{k+1}-\lambda_{k} & \geq \lambda_{1}-\lambda_{0}=\left(9 / b^{2}\right)\left(d_{1}-d_{0}\right)=\left(9 / b^{2}\right) \sin ^{2}\left(\frac{\pi}{2 n+2}\right) \\
& \geq\left(9 / b^{2}\right)\left(\frac{1}{n+1}\right)^{2} \geq\left(9 / b^{2}\right)\left(b^{2} / 9\right) \geq 1, \quad k=0,1, \ldots, n,
\end{aligned}
$$

and it follows from (2.1) that

$$
\max _{-b / 9 \leq t \leq b / 9}\left|G_{b}(t)\right| \leq \frac{6}{b}\left(\frac{e}{3}\right)^{3 / b} \leq 12 \exp (-c / b)
$$

with an absolute constant $c>0$. If $b \leq 3$ is not of the form $b=\frac{3}{n+1}$ with an even non-negative integer $n$, then we choose the largest even integer $n$ such that $b<\beta:=\frac{3}{n+1}$ and the example in the already studied case shows that

$$
\max _{-b / 9 \leq t \leq b / 9}\left|G_{\beta}(t)\right| \leq \max _{-\beta / 9 \leq t \leq \beta / 9}\left|G_{\beta}(t)\right| \leq 12 \exp (-c / \beta) \leq 12 \exp \left(-c^{*} / b\right)
$$

with an absolute constant $c^{*}:=c / 3>0$. Choosing $b=9 a \leq 3$ we obtain our claim for all $a \in(0,1 / 3]$.

Remark 2.6. Using a slightly better lower bound for $n$ !, by a straightforward modification of Remark 2.5 one can see that

$$
\inf _{g} \max _{t \in[-a, a]}|g(t)| \leq \exp \left(\frac{-1}{e^{2} a}\right)\left(\frac{2}{e}+e a\right) \sqrt{\frac{e^{2}+1 / a}{2 \pi}}
$$

for all $a \in\left(0,1 /\left(2 e^{2}\right)\right]$, where the infimum is taken for all exponential sums $g$ of the form

$$
g(t)=\sum_{k=0}^{n+1} a_{k} e^{i \lambda_{k} t}
$$

with $0=\lambda_{0}<\lambda_{1}<\cdots<\lambda_{n+1}$ satisfying the gap condition

$$
\lambda_{k+1}-\lambda_{k} \geq 1, \quad k=0,1, \ldots, n
$$

( $n=2,4, \ldots$ can be arbitrary) and with $a_{k}$ as in Remark 2.5. 
To see this let $n$ be even and consider

$$
\widetilde{g}_{n}(z):=1-z^{\widetilde{\lambda}_{n+1}}+2 \sum_{k=1}^{n}(-1)^{k} z^{\widetilde{\lambda}_{k}}
$$

where $\widetilde{\lambda}_{k}$ are defined as

$$
\tilde{\lambda}_{k}:=\csc ^{2}\left(\frac{\pi}{2 n+2}\right) \sin ^{2}\left(\frac{k \pi}{2 n+2}\right), \quad k=1, \ldots, n,
$$

and satisfy the gap condition $\widetilde{\lambda}_{k+1}-\widetilde{\lambda}_{k} \geq 1$ for $k=1,2, \ldots, n$. Notice that the rescaled timings satisfy $\widetilde{\lambda}_{k}=d_{k} / d_{1}$, where the numbers $d_{k}$ are defined in Remark 2.5. Let $a:=$ $e^{-2} / n$ and define $F_{a}(t):=\widetilde{g}_{n}\left(e^{i t}\right)$. Following the argument given in Remark 2.5, we can show that

$$
\left|F_{a}(t)\right| \leq \exp \left(\frac{-1}{e^{2} a}\right)\left(\frac{2}{e}+e a\right) \sqrt{\frac{e^{2}+1 / a}{2 \pi}}
$$

for all $|t| \leq a$. Notice that the right hand side of the above inequality is an increasing function of $a$. If $a$ cannot be written as $e^{-2} / n$ for an even integer $n$, we may simply use the smallest even integer $n$ such that $a>\alpha:=e^{-2} / n$ whenever $0<a<1 /\left(2 e^{2}\right)$. Thus

$$
\max _{-a \leq t \leq a}\left|F_{\alpha}(t)\right| \leq \max _{-\alpha \leq t \leq \alpha}\left|F_{\alpha}(t)\right| \leq \exp \left(\frac{-1}{e^{2} a}\right)\left(\frac{2}{e}+e a\right) \sqrt{\frac{e^{2}+1 / a}{2 \pi}} .
$$

Remark 2.7 Finding a polynomial $\sum_{j=1}^{n} a_{j} z^{\lambda_{j}}$ with $a_{j} \in\{-1,1\}$, integer exponents $0<\lambda_{1}<\lambda_{2}<\cdots<\lambda_{2 n}$, and with a zeros at 1 of multiplicity at least $n$ is closely related to Wright's conjecture (1934) on ideal solutions of the Prouhet-Tarry-Escott Problem. This seems extremely difficult to settle. See [1, Chapter 11] about the history of this problem. However, as P. Borwein writes it in [1, p. 87], heuristic arguments suggest that Wright's conjecture should be false.

\section{LEMMAS}

To prove Theorem 2.2 we modify the proof given in [2] in the case where $\lambda_{j}=j$ for each $j$. We need the lemmas.

Lemma 3.1. Let $0<a \leq \pi$ and $N \geq 1$. For every $g \in \mathcal{S}_{N}^{\mu}$ with $\left|g\left(\frac{1}{4 N e^{\mu}}\right)\right| \geq 4^{-(\mu+1)}$ there is a value $b \in\left[\frac{1}{2}, \frac{3}{4}\right]$ such that $|g(b)| \geq c_{2}>0$, where $c_{2}$ depends only on $N \geq 1$ and $\mu \geq 0$.

Proof of Lemma 3.1. The proof is a standard normal family argument. Suppose the lemma is not true for some $N \geq 1$ and $\mu \geq 0$. Then there is a sequence $\left(g_{n}\right)$ such that $g_{n} \in \mathcal{S}_{N}^{\mu}$, $\left|g_{n}\left(\frac{1}{4 N e^{\mu}}\right)\right|=4^{-(\mu+1)}$ and

$$
\lim _{n \rightarrow \infty} K_{n}=0, \quad K_{n}:=\max _{z \in\left[\frac{1}{2}, \frac{3}{4}\right]}\left|g_{n}(z)\right|, \quad n=1,2, \ldots
$$

Then there is a subsequence of $\left(g_{n}\right)$, without loss of generality we may assume that this is $\left(g_{n}\right)$ itself, that converges to a function $g \in \mathcal{S}_{N}^{\mu}$ locally uniformly on every compact subset of

$$
D^{+}:=\{z \in \mathbb{C}:|z|<1, \operatorname{Re}(z)>0\} .
$$

Now $g(z)=0$ for all $z \in\left[\frac{1}{2}, \frac{3}{4}\right]$ while $g\left(\frac{1}{4 N e^{\mu}}\right)=4^{-(\mu+1)}$. This contradicts the Unicity Theorem. 
Lemma 3.2. Let $0<a \leq \pi . b \in\left[\frac{1}{2}, \frac{3}{4}\right]$, and $N \geq 1$. Let $\Gamma_{a, b}$ be the circle centered at $b$ with diameter $[2 b-\cos (a / 8), \cos (a / 8)]$. Let $I$ be the subarc of $\Gamma_{a, b}$ with length $\ell(I) \geq c_{3} a$ with midpoint $\cos (a / 8)$ on the real line, where $c_{3} \in(0,1]$. Then there is a constant $c_{4}>0$ depending only on $N, \mu, c_{2}$, and $c_{3}$ such that

$$
\max _{z \in I}|g(z)| \geq \exp \left(-c_{4} / a\right)
$$

for every $g \in \mathcal{S}_{N}^{\mu}$ with $|g(b)| \geq c_{2}>0$.

Proof of Lemma 3.2. Let $2 m \geq 4$ be the smallest even integer not less than $4 \pi /\left(c_{3} a\right)$. Let

$$
\xi:=\exp \left(\frac{\pi i}{m}\right)
$$

be the first $(2 m)$-th root of unity. We define $2 m$ equally spaced points on $\Gamma_{a, b}$ by

$$
\eta_{k}:=b+(\cos (a / 8)-b) \xi^{k}, \quad k=0,1, \ldots, 2 m-1
$$

Then there is a constant $c_{5}>0$ depending only on $c_{3}$ such that

$$
1-|z| \geq c_{5}(k a)^{2}, \quad k=1,2, \ldots m-1
$$

whenever $z$ is on the smaller subarc of the circle $\Gamma_{a, b}$ with endpoints $\eta_{k}$ and $\eta_{k+1}$ or with endpoints $\eta_{2 m-k}$ and $\eta_{2 m-k-1}$, respectively. We define the function

$$
h(z):=\prod_{j=0}^{2 m-1} g\left(b+(\cos (a / 8)-b) \xi^{j}(z-b)\right)
$$

If $g \in \mathcal{S}_{N}^{\mu}$, then

$$
\begin{aligned}
\max _{z \in \Gamma_{a, b}}|h(z)| & \leq\left(\prod_{k=1}^{m-1}\left(N\left(\frac{1}{c_{5}(k a)^{2}}\right)^{\mu}\right)\right)^{2}\left(\max _{z \in I}|g(z)|\right)^{2} \\
& \leq\left(\frac{1}{c_{5} a}\right)^{(4 m-4) \mu} \frac{N^{2 m-2}}{((m-1) !)^{4 \mu}}\left(\max _{z \in I}|g(z)|\right)^{2} \\
& \leq\left(\frac{m}{2 \pi c_{3} c_{5}}\right)^{(4 m-4) \mu}\left(\frac{e}{m-1}\right)^{(4 m-4) \mu} N^{2 m-2}\left(\max _{z \in I}|g(z)|\right)^{2} \\
& \leq e^{4 \mu} N^{2 m-2}\left(\frac{e}{2 \pi c_{3} c_{5}}\right)^{(4 m-4) \mu}\left(\max _{z \in I}|g(z)|\right)^{2} \\
& \leq \exp \left(c_{6} / a\right)\left(\max _{z \in I}|g(z)|\right)^{2}
\end{aligned}
$$


with a constant $c_{6}>0$ depending only on $N, \mu$, and $c_{3}$. Now the Maximum Principle yields that

$$
|g(b)|^{2 m}=|h(b)| \leq \max _{z \in \Gamma_{a, b}}|h(z)| \leq \exp \left(c_{6} / a\right)\left(\max _{z \in I}|g(z)|\right)^{2} .
$$

Since $2 m \leq 2+4 \pi /\left(c_{3} a\right)$ and $|g(b)| \geq c_{2}$, we obtain

$$
\left(\max _{z \in I}|g(z)|\right)^{2} \geq \exp \left(-c_{6} / a\right)|g(b)|^{2 m} \geq \exp \left(-c_{6} / a\right)\left(c_{2}\right)^{2 m} \geq \exp \left(-2 c_{4} / a\right)
$$

with a constant $c_{4}>0$ depending only on $N, \mu, c_{2}$, and $c_{3}$.

Lemma 3.3. Let $0<a<\pi, N \geq 1$, and $\mu=1,2, \ldots$ Let $A:=\left\{e^{i t}: t \in[-a / 2, a / 2]\right\}$. There is a constant $c_{7}>0$ depending only on $N$ and $\mu$ such that

$$
\int_{A}|f(z)||d z| \geq \exp \left(-c_{7} / a\right)
$$

for every $f \in \mathcal{S}_{N}^{\mu}$ that is analytic on the arc $A$ and satisfies $\left|f\left(\frac{1}{4 N e^{\mu}}\right)\right| \geq \frac{1}{2}$.

To prove Lemma 3.3 we need the following.

Lemma 3.4. Let $w_{1} \neq w_{2} \in \mathbb{C}$ and let $z_{0}:=\frac{1}{2}\left(w_{1}+w_{2}\right)$. Assume that $J_{1}$ is an arc that connects $w_{1}$ and $w_{2}$. Let $J_{2}$ be the arc that is the symmetric image of $J_{1}$ with respect to the $z_{0}$. Let $J:=J_{1} \cup J_{2}$ be positively oriented. Suppose that $g$ is an analytic function inside and on $J$. Suppose that the region inside $J$ contains the disk centered at $z_{0}$ with radius $\gamma>0$. Let $|g(z)| \leq K$ for $z \in J_{2}$. Then

$$
\left|g\left(z_{0}\right)\right|^{2} \leq(\pi \gamma)^{-1} K \int_{J_{1}}|g(z)||d z|
$$

Proof of Lemma 3.4. Applying Cauchy's integral formula with

$$
G(z):=g\left(z_{0}+\left(z-z_{0}\right)\right) g\left(z_{0}-\left(z-z_{0}\right)\right)
$$

on $J$, we obtain

$$
\begin{aligned}
\left|g\left(z_{0}\right)\right|^{2} & =\left|G\left(z_{0}\right)\right|=\left|\frac{1}{2 \pi i} \int_{J} \frac{G(z) d z}{z-z_{0}}\right| \\
& =\frac{2}{2 \pi}\left|\int_{J_{1}} \frac{G(z) d z}{z-z_{0}}\right| \leq \frac{1}{\pi} \int_{J_{1}} \frac{|G(z)||d z|}{\left|z-z_{0}\right|} \\
& =\frac{1}{\pi} \int_{J_{1}} \frac{\left|g\left(z_{0}+\left(z-z_{0}\right)\right) g\left(z_{0}-\left(z-z_{0}\right)\right)\right||d z|}{\left|z-z_{0}\right|} \\
& \leq(\pi \gamma)^{-1} K \int_{J_{1}}|g(z)||d z| .
\end{aligned}
$$


Proof of Lemma 3.3. Without loss of generality we may assume that $\ell(A)=a \leq \pi / 2$. Suppose $f \in \mathcal{S}_{N}^{\mu}$ and $\left|f\left(\frac{1}{4 N e^{\mu}}\right)\right| \geq \frac{1}{2}$. Let the region $H_{a}$ be defined by

$$
H_{a}:=\left\{z=r e^{i \theta}: \cos (a / 4)<r<\cos (a / 8),-a / 4<\theta<a / 4\right\} .
$$

Associated with $a \in(0,1]$ and $b \in\left[\frac{1}{2}, \frac{3}{4}\right]$ (the choice of $b$ will be specified later), let $\Gamma_{a, b}$ be the circle as in Lemma 3.2. It is easy to see that the arc $I:=\Gamma_{a, b} \cap H_{a}$ has length greater than $c_{3} a$ with an absolute constant $c_{3}>0$. Let $f \in \mathcal{S}_{N}^{\mu}$. Let $z_{0} \in I \subset H_{a}$ be chosen so that

$$
\left|f\left(z_{0}\right)\right|=\max _{z \in I}|f(z)| \text {. }
$$

Also, we can choose $w_{1} \in A$ and $w_{2} \in A$ such that $z_{0}=\frac{1}{2}\left(w_{1}+w_{2}\right)$. Let $J_{1}$ be the arc connecting $w_{1}$ and $w_{2}$ on the unit circle. Note that $J_{1}$ is a subarc of $A$ of length at least $a / 4$. Let $J_{2}$ be the arc which is the symmetric image of $J_{1}$ with respect to the line segment connecting $w_{1}$ and $w_{2}$. Let

$$
g(z):=4^{-\mu}\left(\left(z-w_{1}\right)\left(z-w_{2}\right)\right)^{\mu} f(z) .
$$

It is elementary geometry again to show that

$$
|g(z)| \leq \frac{4^{-\mu} N\left|\left(z-w_{1}\right)\left(z-w_{2}\right)\right|^{\mu}}{(1-|z|)^{\mu}} \leq \frac{4^{-\mu} N 2^{\mu}}{\sin ^{\mu}(a / 8)}=\frac{2^{-\mu} N}{\sin ^{\mu}(a / 8)}, \quad z \in J_{2} .
$$

By Lemma 3.4 we obtain

$$
\left|g\left(z_{0}\right)\right|^{2} \leq\left(\pi(1-\cos (a / 8))^{-1} \frac{N 2^{-\mu}}{\sin ^{\mu}(a / 2)} \int_{J_{1}}|g(z)||d z|\right.
$$

Observe that $f \in \mathcal{S}_{N}^{\mu}$ implies $g \in \mathcal{S}_{N}^{\mu}$. Also, since $N \geq 1, \mu \geq 1$, and $\left|f\left(\frac{1}{4 N e^{\mu}}\right)\right| \geq \frac{1}{2}$, we have

$$
\begin{aligned}
\left|g\left(\frac{1}{4 N e^{\mu}}\right)\right| & \geq 4^{-\mu}\left(1-\frac{1}{4 N e^{\mu}}\right)^{2 \mu}\left|f\left(\frac{1}{4 N e^{\mu}}\right)\right| \geq 4^{-\mu}\left(1-\frac{1}{8 \mu}\right)^{2 \mu} \frac{1}{2} \geq 4^{-\mu}\left(\frac{7}{8}\right)^{2} \frac{1}{2} \\
& \geq 4^{-(\mu+1)} .
\end{aligned}
$$

Hence, by Lemma 3.1 , we can pick $b \in\left[\frac{1}{2}, \frac{3}{4}\right]$ so that $|g(b)| \geq c_{2}$ with an absolute constant $c_{2}>0$ depending only on $N$ and $\mu$. Now we can deduce from Lemma 3.2 that

$$
\left|g\left(z_{0}\right)\right| \geq \exp \left(-c_{4} / a\right)
$$

Combining this with (3.1) and $J_{1} \subset A$ gives

$$
\begin{aligned}
\int_{A}|f(z)||d z| & \geq \int_{A}|f(z)||d z| \geq \int_{J_{1}}|g(z)||d z| \\
& \geq \pi(1-\cos (a / 8)) \frac{2^{\mu} \sin ^{\mu}(a / 8)}{N}\left|g\left(z_{0}\right)\right|^{2} \\
& \geq \exp \left(-c_{1} / a\right)
\end{aligned}
$$

with a constant $c_{1}>0$ depending only on $N$ and $\mu$. 


\section{Proof of the theorems}

Proof of Theorem 2.2. Let $f \in \mathcal{K}_{M}^{\mu}(\Lambda)$, where the exponents $\lambda_{j} \in \mathbb{R}$ satisfy

$$
\lambda_{0}=0, \quad \lambda_{j} \geq j>0, \quad j=1,2, \ldots
$$

Then $f \in \mathcal{S}_{M \mu !}^{\mu+1}$ and $f$ is analityc on the arc $A$. Also, if

$$
\left|z_{0}\right| \leq \frac{1}{4 M(\mu+1) ! e^{\mu+1}}
$$

then

$$
\begin{aligned}
\left|f\left(z_{0}\right)\right| & \geq 1-M \sum_{j=1}^{\infty} \lambda_{j}^{\mu}\left(\frac{1}{4 M(\mu+1) ! e^{\mu+1}}\right)^{\lambda_{j}} \geq 1-\frac{M}{4 M(\mu+1) !} \sum_{j=1}^{\infty}\left(\frac{j}{e^{j}}\right)^{\mu+1} \\
& \geq 1-\frac{1}{4} \sum_{j=1}^{\infty}\left(\frac{j}{e^{j}}\right) \geq 1-\frac{1}{4} \sum_{j=1}^{\infty}\left(\frac{j}{2^{j}}\right) \geq 1-\frac{2}{4} \geq \frac{1}{2} .
\end{aligned}
$$

So the assumptions of Lemma 3.3 are satisfied with $\mu$ replaced by $\mu+1$ and $N$ replaced by $M(\mu+1)$ !, and the theorem follows from Lemma 3.3.

Theorem 2.1 is an obvious consequence of Theorem 2.2.

\section{ACKNOWLEDGMENTS}

Work at Dartmouth was supported by the National Science Foundation under grant number PHY-0903727.

\section{REFERENCES}

1. P. Borwein, Computational Excursions in Analysis and Number Theory, Springer, New York, 2002.

2. P. Borwein and T. Erdélyi, Littlewood-type problems on subarcs of the unit circle, Indiana Univ. Math. J. 46 (1997), 1323-1346.

3. P. Borwein and T. Erdélyi, Polynomials and Polynomial Inequalities, Springer-Verlag, New York, N.Y., 1995.

4. P. Borwein, T. Erdélyi, and G. Kós, Littlewood-type problems on [0,1], Proc. London Math. Soc. (3) 79 (1999), 22-46.

5. R.A. DeVore and G.G. Lorentz, Constructive Approximation, Springer-Verlag, Berlin, 1993.

6. K. Khodjasteh, T. Erdélyi, and L. Viola, Limits on preserving quantum coherence using multipulse control (to appear).

7. S. Güntürk, Approximation by power series with \pm 1 coefficients., Int. Math. Res. Not. 26 (2005), 1601-1610.

8. S.V. Konyagin, On a problem of Littlewood, Mathematics of the USSR, Izvestia 18 (1981), 205-225. 
9. S. Konyagin, On a question of Pichorides, C.R. Acad. Sci. Paris Sér I Math. 324 (1997), 385388.

10. O.C. McGehee, L. Pigno, and B. Smith, Hardy's inequality and the $L_{1}$ norm of exponential sums, Ann. of Math. 113 (1981), 613-618.

11. S.K. Pichorides, Notes on trigonometric polynomials., in: Conference on harmonic analysis in honor of Antoni Zygmund, Vol. I (Chicago, Illinois, 1981), 84-94, Wadsworth Math. Ser., Wadsworth, Belmont, Calif., 1983..

12. G.S. Uhrig, Phys. Rev. Lett. 98, 100504 (2007).

13. L. Viola, E. Knill, and S. Lloyd, Phys. Rev. Lett. 82, 2417 (1999).

Department of Mathematics, Texas A\&M University, College Station, Texas 77843

E-mail address: terdelyi@math.tamu.edu

Department of Physics and Astronomy, Dartmouth College, 6127 Wilder laboratory, HANOVER, NH 03755

E-mail address: kaveh.khodjasteh@dartmouth.edu

Department of Physics and Astronomy, Dartmouth College, 6127 Wilder laboratory, HANOVER, NH 03755

E-mail address: Lorenza.Viola@Dartmouth.edu 